\title{
MEIOFAUNA DA ÁREA RECIFAL DA BAÍA DE TAMANDARÉ (PERNAMBUCO, BRASIL)
}

\author{
Grácia Maria Bártholo MARANHÃO \\ Doutoranda do P.P-G-Oceanografia da UFPE \\ Verônica da FONSÊCA-GENEVOIS
}

Departamento de Zoologia da UFPE

José Zanon de Oliveira. PASSAVANTE

Departamento de Oceanografia da UFPE

\begin{abstract}
RESUMO
A meiofauna foi coletada mensalmente de março a dezembro de 1991, em uma área recifal da baía de Tamandaré, junto a foz do estuário do rio Mamucaba, em regime de baixa mar. Quatro estações foram prospectadas: a primeira na parte interna do recife; a segunda na poça rasa de maré; a terceira na poça média de maré, com dois pontos um com muita luminosidade (cerca de $0,3 \mathrm{~m}$ de profundidade) e o outro com pouca luminosidade (cerca de $0,5 \mathrm{~m}$ de profundidade) e a quarta estação no encrave com três pontos um com luminosidade intensa (cerca de 1,0m de profundidade), o outro com luminosidade intermediária (cerca de 2,0m de profundidade) e o último com pouca luminosidade (cerca de 3,0m de profundidade). As amostras biosedimentológicas para análise da meiofauna foram coletadas manualmente ou por mergulho em apnéia e acondicionada em garrafas plásticas de $500 \mathrm{ml}$, de cada ambiente recifal também foram coletados sedimentos, servindo às análises granulométricas. Para a obtenção da meiofauna, os sedimentos coletados, sofreram lavagens em peneiras geológicas de 0,044mm e 1,0mm, sob água corrente à pressão graduada, sendo utilizado o material retido na peneira com abertura de malha de $0,044 \mathrm{~mm}$. Este material era vertido em placa de Petri, onde três subamostras foram retiradas e analisadas em placa de Dollfus. A meiofauna esteve representada por Turbellaria, Nematoda, Annelida Polychaeta, Archiannelida, Copepoda Harpacticoidea, Copepoda Cyclopoidea, Ostracoda e Acarina. Em termos espaciais a meiofauna apresentou distribuição contínua variando entre 3 e 6 grupos. A meiofauna apresentou diferenças entre os períodos seco e chuvoso, sendo o primeiro menos variável taxonomicamente. A composição quantitativa da meiofauna obteve seu máximo na poça média com luminosidade intensa $(0,5 \mathrm{~m})$, cuja granulometria é dominada por areia média, com pico máximo de $885 \mathrm{ind} .500 \mathrm{ml}^{-1}$ em setembro. O coeficiente de Spearman na poça rasa mostrou o maior número de correlações positivas entre os taxa da comunidade. Na granulometria dominou areia grossa a média. Nas poças de menor profundidade a meiofauna apresentou uma maior variação taxonômica, não existindo variação sazonal em relação à granulometria dos sedimentos, a temperatura e aos parâmetros químicos. A meiofauna recifal apresentouse mais estável do que as de linha de praias.

Palavras chave: Meiofauna, Baía de Tamandaré, Recifes, Tropical
\end{abstract}

\section{ABSTRACT \\ Meiofauna of Tamandaré Bay Reef Area (Pernambuco, Brazil)}

Trab. Oceanog. Univ. Fed. PE, Recife, 28(1): 47- 59, 2000. 
Studies about the recifal meiofauna were carried out at Tamandaré (Pernambuco - Brazil) from March to December 1991. Sampling were made within the first reef line on a southern area of Tamandaré bay, near Mamucaba River estuary. Four stations were sampled during low tide: the first one in the inner reef area, the second one in a small tide pools, the third in a deeper tide pools with two different conditions (high light intensity - 0.3m depth and low light intensity - 0.5m depth) and the fourth in larger pool with three different conditions (high light intensity $-1.0 \mathrm{~m}$ depth, mean light intensity - 2.0m depth and low light intensity - 3.0m depth). Samples for meiofauna and sediments were hand-collected through free dives. Meiofauna was extracted from the sediment through successive washes and retained on sieves within the interval of 0.044 to $1.0 \mathrm{~mm}$ mesh sizes. Three subsamples were used to count the meiofauna on a Dolfus plates. Meiofauna was composed by Turbellaria, Nematoda, Annelida Polychaeta, Annelida Archiannelida, Copepoda Harpacticoida, Copepoda Cyclopoida, Ostracoda and Acari. Five to six groups presented continuous spatial distribution. Differences on the meiofauna composition were observed, mainly between the rainy and dry seasons. The dry season is more stable. Maximum densities (885ind. $500 \mathrm{ml}^{-1}$ ) were observed on tide pools around $0.5 \mathrm{~m}$ depth with high light intensity and a sandy sediment. Spearman Correlation coefficient showed a greater number of significant community correlation at low depth tide pools, where sediment was composed both by mean and coarse sand. At low depths, meiofauna presented a greater temporal instability, with no relation to temperature, sediment granulometry and chemical parameters. The meiofauna community in the studied reef was clearly more stable than those from sandy shores.

Key words: Meiofauna, Tamandaré Bay, Reefs, Tropical

\section{INTRODUÇÃO}

A meiofauna foi definida por Mare (1942) em função do seu habitat e de sua dimensão. Trata-se do conjunto de metazoários cujos limites corpóreos estão compreendidos entre os intervalos de 40 a 500 micrômetros, ocupando os interstícios dos sedimentos, ou utilizando outros substratos da superfície sedimentar de ambientes aquáticos (Dye e Furstenberg, 1978; Reise e Ax , 1979; Moore, 1979; ReunaudMornant et al., 1984).

Para caracterização da meiofauna aspectos relevantes têm sido abordados, de modo que a seleção aqui empregada diz respeito aos metazoários retidos em peneiras com abertura de malha entre 0,044mm a 1,0mm (Medeiros, 1989).

$\mathrm{O}$ estudo da comunidade meiofaunística vem crescendo rapidamente por ter relevância em avaliar o estresse do meio, uma vez que é entendida como "partícula viva do sedimento" guardando características deixadas pelos agentes poluidores. Estes organismos demonstram seu sucesso adaptativo, por possuírem a capacidade de recolonizar o ambiente marinho muito rapidamente, sobretudo, porque são, na grande maioria, organismos holobênticos, de ciclo de vida curto, dispersando-se por imposições hidrodinâmicas (Renaud-Mornant et al.,1984).

Estudos sobre a meiofauna de sedimentos recifais foram realizados para Bahamas (Renaud-Mornant e Gourbault, 1981, 1984), para as Antilhas (Gourbault et al., 1985) e para Costa Rica (Guzmá, 1987) enfocando populações de Nematoda e Tardigrada. No entanto, os autores referem-se aos sedimentos, seja intermareal ou de 
recifes coralinos submersos, em condições diferentes da região na qual foi realizada o presente trabalho.

Os "beachrocks" que ocorrem paralelos ao litoral formam cordões de arenito que funcionam como corredores para que as águas circulem na zona costeira, retendo-as entre os cordões de arenito e a linha de praia (Maÿal e Barone, no prelo).

Maida e Ferreira (no prelo) historiaram os recifes brasileiros desde 1828 e ressaltam a importância da investigação realizada por Laborel (1967). Ferreira et al. (1995) indicaram que os recifes de Tamandaré fazem parte da formação recifal que se estende por cerca de 3.000 quilômetros na costa Nordeste do Brasil.

Dentre os estudos realizados na região de Tamandaré a partir da década de 80, ressalta-se aqueles referentes à fauna bêntica (Mayal e Amaral, 1990; Amaral,1992; Pena, 1998; Amaral, 1994; Coelho e Ramos Porto, 1995; Lira e Fonsêca-Genevois, 1998; Maÿal e Barone, no prelo); aos peixes associados aos recifes (Ferreira et al., 1995); ao microzooplâncton (Galvão e Lins da Silva, 1994, 1996a, 1996b); ao microfitoplâncton (Galvão e Lins da Silva, 1995), a biomassa e produção primária (Moura, 1991) e ao zooplâncton (Porto Neto et al., 2000).

Em relação a meiofauna, vários estudos de cunho geral foram realizados em sedimentos costeiros (Carvalho et al., 1992; Fonsêca-Genevois e Carvalho, 1992; Fonsêca-Genevois, 1992; Fonsêca-Genevois et al., 1992; Silva, 1997; Teixeira, 1991; Maranhão e Fonsêca-Genevois, 1991, 1992, 1993; Fonsêca-Genevois e Maranhão, 1996).

No sentido de contribuir para o conhecimento da meiofauna em ambientes recifais, realizou-se o presente estudo, cujo objetivo foi conhecer a composição e densidade em escala temporal e espacial e a relação com o tipo de sedimento.

\section{MATERIAL E MÉTODOS}

A Baía de Tamandaré $\left(8^{\circ} 44^{\prime} 23^{\prime \prime}\right.$ e $8^{\circ} 44^{\prime} 41^{\prime \prime}$ Lat. S e $35^{\circ} 7^{\prime} 29^{\prime \prime}$ e $35^{\circ} 2^{\prime}$ 28” Long. W) sofre grande influência dos rios Mamucabinha e Ilhetas, tendo uma profundidade média cerca de 7 a $8 \mathrm{~m}$. Encontra-se também nesta região formações de arenito, recifes superficiais, com espessuras inferiores a $1 \mathrm{~m}$, assentados sobre cordões de arenito de praia (Mayal e Barone, no prelo), que ficam expostos na baixa mar, totalmente isolados (Fig. 1).

Em Tamandaré, a meiofauna foi coletada mensalmente de março a dezembro de 1991, na linha recifal da baía, nas proximidades do rio Mamucaba, no regime de baixa mar, sendo escolhidas quatro estações de coletas : a primeira na parte interna do recife; a segunda na poça rasa de maré; a terceira na poça média de maré, com dois pontos um com pouca luminosidade (cerca de $0,3 \mathrm{~m}$ de profundidade) e o outro com luminosidade intensa (cerca de $0,5 \mathrm{~m}$ de profundidade) e a quarta estação no encrave com três pontos um com luminosidade intensa (cerca de 1,0m de profundidade), outro com luminosidade intermediária (cerca de 2,0m de profundidade) e o último com pouca luminosidade (cerca de 3,0m de profundidade) 


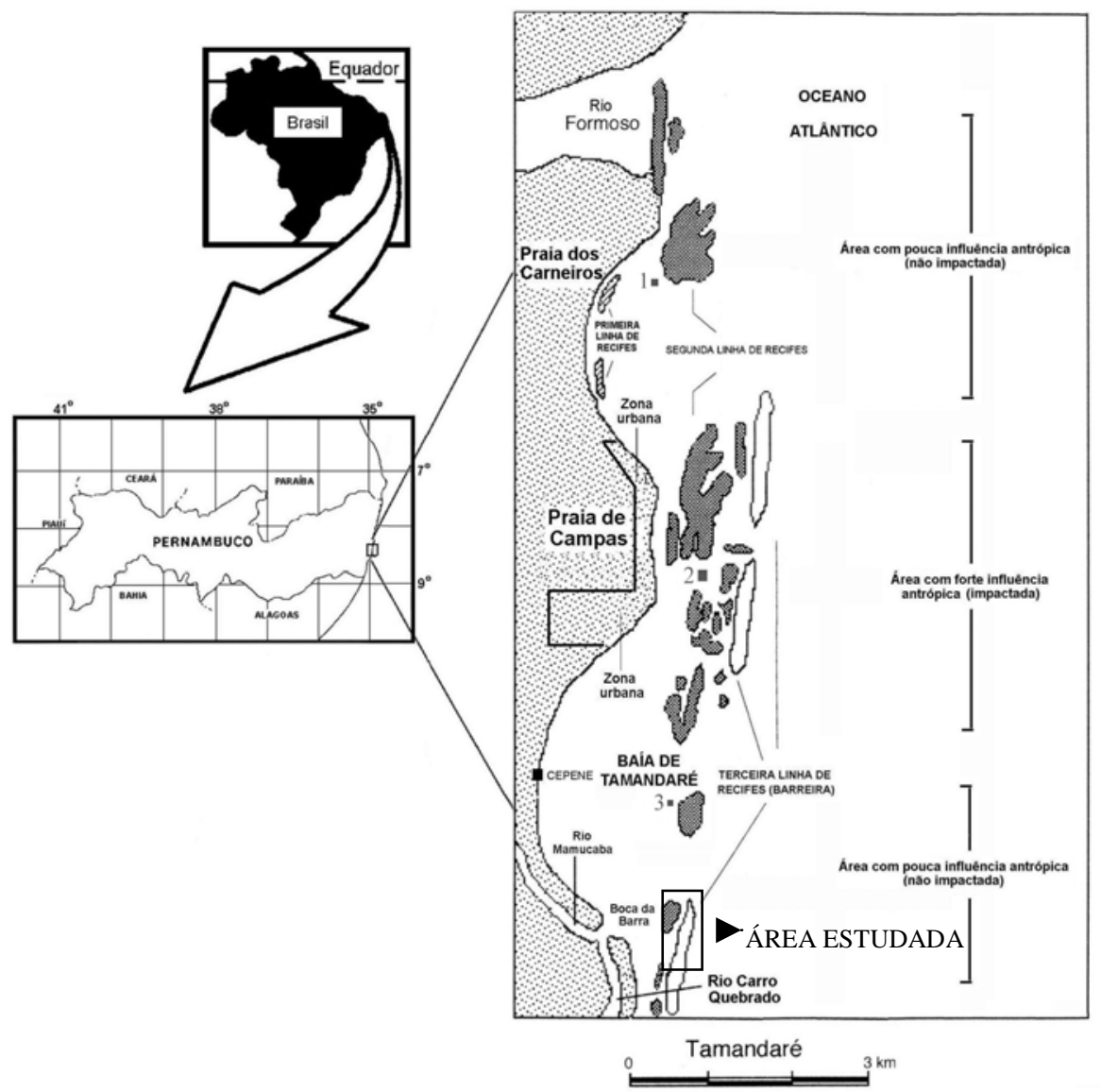

Figura 1 - Área estudada (Fonte: Porto Filho et al., 2000)

As amostras foram coletadas, manualmente com auxílio de um recipiente plástico e em maiores profundidades por mergulho em apnéia e acondicionadas em garrafas plásticas de $500 \mathrm{ml}$, etiquetadas e fixadas com formol salino a $4 \%$. Em cada estação foram também coletados sedimentos servindo às análises granulométricas, água intersticial e meiofaunística.

As amostras para triagem da meiofauna sofreram lavagens em peneiras geológicas de 0,044mm; 0,250mm e 1,00mm, sob água corrente à pressão graduada. Em seguida, o material retido na peneira com abertura de malha de $0,044 \mathrm{~mm}$, estes considerados pertencentes à meiofauna verdadeira, foi vertido em placa de Petri. Destas três subamostras foram retiradas e analisadas em placas de Dollfus, sendo esta analisada em seus duzentos quadrados, a fim de separar a meiofauna a nível dos grandes grupos 
zoológicos de acordo com a metodologia de rotina descrita por Fonsêca-Genevois (1987) .

Os taxa foram separados a nível de grandes grupos zoológicos, com o auxílio de estereomicróspio de marca Zeiss e foram armazenados em recipientes de vidro, contendo formol a $4 \%$ e glicerina. Este material encontra-se catalogado no Laboratório de Invertebrados Aquáticos no Departamento de Zoologia da Universidade Federal de Pernambuco.

Foi realizado teste não paramétrico através do "Coeficiente de Spearman", o qual foi utilizado para se ter uma idéia das correlações entre os componentes da comunidade de cada um dos ambientes prospectados.

\section{RESULTADOS}

\section{Composição qualitativa da meiofauna}

A comunidade meiofaunística dos diversos ambientes recifais esteve composta pelos seguintes grupos: Turbellaria, Nematoda, Annelida Polychaeta, Archiannelida, Copepoda Harpacticoida, Copepoda Cyclopoida, Ostracoda e Acarina, aqui representada em ordem filogenética (Barnes,1984). No entanto, a dominância de cada grupo apresentou variações espaciais e temporais.

De acordo com os diversos locais amostrados, a meiofauna esteve representada na parte interna dos recifes por Turbellaria, Nematoda, Copepoda Harpacticoidea e Copepoda Cyclopoidea; na poça rasa, por: Turbellaria, Nematoda, Annelida Polychaeta, copepodito, Copepoda Harpacticoida (fêmeas ovígeras) e Ostracoda; na porção de pouca luminosidade da poça média, por Turbellaria, Nematoda e Annelida Polychaeta; na porção com luminosidade intensa da poça média $(0,3 \mathrm{~m})$, por Nematoda, Annelida Polychaeta, Copepoda Harpacticoida e Copepoda Cyclopoidea; no encrave, com luminosidade intensa $(1,0 \mathrm{~m})$, por Turbellaria, Nematoda, Annelida Polychaeta, Copepoda Harpacticoidea, adultos e fêmeas ovígeras e Copepoda Cyclopoidea; nas porções de luminosidade intermediária e de pouca luminosidade $(3,0 \mathrm{~m})$ do Encrave por Turbellaria , Nematoda e Copepoda Harpacticoidea.

\section{Abundância relativa}

$\mathrm{Na}$ parte interna dos recifes os Nematoda foram mais abundantes. Na poça rasa os Copepoda dominaram a comunidade durante todos os meses do ano. Na poça média com pouca luminosidade os Copepoda Harpacticoida dominaram sobre os outros grupos, exceto em março e junho, quando os Nematoda atingiram $46 \%$ do total e em junho, quando foi registrado $44 \%$ de Turbellaria. Na porção de luminosidade intensa, verificou-se que os Copepoda chegam a perfazer o percentual de $75 \%$ no mês de agosto, sendo 65\% referente aos Harpacticoida. Copepoda foi superado por Ostracoda em setembro, com 37\% e em junho por Annelida Polychaeta, que alcançaram 31\%. Na porção de luminosidade intensa do encrave $(1,0 \mathrm{~m})$ Nematoda foi mais abundante em quase todos os meses, com mesma abundância de Copepoda em novembro e superado por Ostracoda em julho (34\%). Na porção de luminosidade intermediária Nematoda dominou durante os cinco meses, com valor máximo de $91 \%$ em maio. Durante três

Trab. Oceanog. Univ. Fed. PE, Recife, 28(1): 47- 59, 2000. 
meses Copepoda atingiu maior concentração, sobretudo em setembro com $46 \%$. Na porção de pouca luminosidade do encrave $(3,0 \mathrm{~m})$, Nematoda chegou a atingir $90 \%$ da comunidade em julho, sendo dominante também em março, abril, junho, setembro, novembro e dezembro. Nos meses restantes a abundãncia máxima foi de Copepoda.

A meiofauna atingiu em função do total de indivíduos 13,95\% na parte interna; 13,45\% na poça rasa; $9,75 \%$ na poça média com pouca luminosidade; $19,56 \%$ na poça média com luminosidade intensa; 16,72\%; na porção com luminosidade intensa do encrave (1,0m); 9,72\%; na porção de luminosidade intermediária (2,0m) e 16,01\%; na porção de pouca luminosidade $(3,0 \mathrm{~m})$.

\section{Densidade}

O somatório anual da meiofauna em todos os ambientes recifais estudados totalizou 23.127 indivíduos, dos quais 39,2\% perfazem o total dos Copepoda nas amostras, enquanto 30,3\% pertencem aos Nematoda .Atribui-se ainda 30,6\% a contribuição dos demais grupos pertencentes à comunidade meiofaunística.

Os Copepoda representaram o primeiro grupo da hierarquia numérica, atingindo densidade média anual de 906,1 ind.500 $\mathrm{ml}^{-1}$. A densidade máxima foi de 1.911 ind.500 $\mathrm{ml}^{-1}$, em outubro, sendo que 92,31\% foi representado pelos Harpacticoida e o restante pelos Cyclopoida. O valor mínimo de 207 ind.500 $\mathrm{ml}^{-1}$ foi observado em maio com percentual de $90,34 \%$ sendo os mais representativos os Harpacticoida. A densidade média da estação seca superou em quase o dobro da estação chuvosa.

A densidade máxima de Harpacticoida ocorreu em outubro com 943 ind.500 $\mathrm{ml}^{-1}$ na porção mais luminosa do encrave. O grupo não foi registrado em junho na poça média. Em relação ao total dos ambientes prospectados, a densidade média foi de 635,2 ind.500 $\mathrm{ml}^{-1}$, sendo $26,98 \%$ referente aos meses de maior pluviometria e 73,02\% correspondente aos meses do período seco.

O maior índice populacional de fêmeas ovígeras de Copepoda Harpacticoidea, ocorreu em outubro na porção mais rasa do encrave com 54 ind.500 $\mathrm{ml}^{-1}$, enquanto nos meses de março, maio, junho, julho e dezembro observou-se a ausência de fêmeas ovígeras nos diversos ambientes estudados.

Copepoda Cyclopoida apresentou valor médio anual de 210,5 ind.500 $\mathrm{ml}^{-1}$ sendo os maiores valores registrados na estação chuvosa. Do total, 66,84\% ocorreu nesta estação, enquanto 33,16\% na estação seca. A densidade máxima foi de 434 ind.500 $\mathrm{ml}^{-1}$ no mês de abril na poça rasa. Não foram registrados representantes em março, junho, setembro, outubro e novembro nos ambientes amostrados. As fêmeas ovígeras ocorreram com máxima densidade em julho com 30 ind.500 $\mathrm{ml}^{-1}$. Observou-se que a maior incidência das fêmeas ocorre na época de maior pluviometria.

Além das fases adultas os Copepoda ocorreram sob forma larvar de náuplios e copepoditos. Os náuplios ocorreram descontinuamente nos ambientes estudados com densidade máxima de 219 ind.500 $\mathrm{ml}^{-1}$ em outubro, valor este no mesmo local de máximo populacional dos Harpacticoida (encrave com maior incidência luminosa). Os copepoditos atingiram o máximo de 225 ind.500 $\mathrm{ml}^{-1}$ na mesma estação e mês. Observou-se que sua contribuição é contínua nos meses estivais.

Nematoda foi o segundo grupo em abundância numérica nos ambientes recifais, atingindo uma densidade média anual de 695,5 ind.500 $\mathrm{ml}^{-1}$ de sedimentos. Seu 
valor máximo foi registrado em setembro com 498 ind.500 $\mathrm{ml}^{-1}$ na parte interna da linha recifal. O valor mínimo foi de 5,0 ind.500 $\mathrm{ml}^{-1}$, em agosto na poça com incidência média luminosa. Dos ambientes prospectados a média dos valores mostram os máximos na parte interna $\left(249,3\right.$ ind. $\left.500 \mathrm{ml}^{-1}\right)$ e na porção mais profunda do encrave (212,6 ind. $500 \mathrm{ml}^{-1}$ ).

A Classe Polychaeta, comparando com os demais Grupos meiofaunísticos apresentou uma distribuição contínua, exceto na estação localizada na parte interna dos recifes, na qual não foi registrada nos meses de março, junho e setembro. A densidade máxima foi de 175 ind. $500 \mathrm{ml}^{-1}$ em novembro na poça com maior intensidade de luz. Fêmeas adultas com bolsas epidérmicas repletas de ovos foram registradas de forma descontínua em baixa densidade. No encrave de profundidade, ocorreu valor máximo de 15 ind. $500 \mathrm{ml}^{-1}$ no mês de dezembro.

Turbellaria apresentou densidades inferiores aos outros grupos, com valor máximo de 74 ind.500 $\mathrm{ml}^{-1}$ em março, na poça média com menor incidência de luminosidade. Na estação de maior incidência luminosa no mês de outubro verificou-se o desaparecimento da população na poça média.

Ostracoda apresentou distribuição descontínua, embora tenha alcançado um percentual de $90 \%$ na poça rasa. A densidade máxima foi de 335 ind. $500 \mathrm{ml}^{-1}$ e ocorreu no encrave, na menor profundidade, no mês de julho. O maior pico ocorreu em setembro, na poça média ( 320 ind. $500 \mathrm{ml}^{-1}$ ).

Archiannelida e Acarina foram os menos densos das amostras estudadas. Archiannelida alcançou densidade máxima de 17 ind. $500 \mathrm{ml}^{-1}$, na poça média com maior intensidade luminosa, no mês de junho. Acarina apresentou densidade máxima de 16 ind. $500 \mathrm{ml}^{-1}$ no mês de maio, na poça média na estação de maior intensidade luminosa.

\section{Freqüência de ocorrência}

Foram muito freqüentes na área estudada ( $>70 \%)$ Nematoda, Copepoda Harpacticoida, Tardigrada, Annelida Polychaeta, Copepoda Cyclopoida, copepodito e Ostracoda. Os demais grupos registrados ocorreram com valores $<40 \%$ (Figura 2).

\section{Análise estatística}

Na parte interna dos recifes foram observadas correlações positivas entre fêmeas ovígeras de Copepoda Harpacticoida e Copepodito $(0,95)$, com Copepoda Harpacticoida com o mesmo estágio de desenvolvimento do grupo $(0,78)$, assim como com ambos os estágios adultos $(0,83)$.

Na poça rasa recifal, Nematoda correlacionou-se positivamente com Copepoda Harpacticoida adulto $(0,82)$, com as fêmeas ovígeras dos Cyclopoidea $(0,70)$ e com Cyclopoida adulto $(0,80)$. Destaca-se que este último mantém fortes correlação com Turbellaria $(0,80)$ e com Copepoda Harpacticoida adulto $(0,83)$

$\mathrm{Na}$ poça média (com pouca luminosidade), foram detectadas correlações positivas entre Copepoda Harpacticoidea adulto e Copepodito $(0,88)$, fêmeas ovígeras e Copepodito $(0,98)$, fêmeas ovígeras com os adultos $(0,79)$ e com Ostracoda $(0,74)$. 


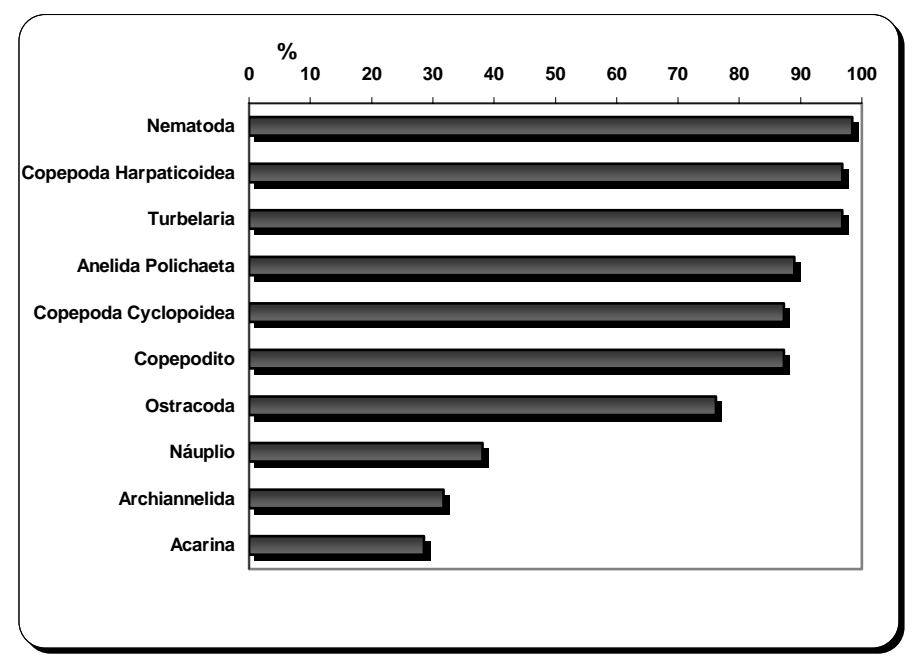

Figura 2 - Freqüência de ocorrência dos organismos meiofaunístico da área recifal de Tamandaré, Pernambuco

Na poça média (com luminosidade intensa), foram extraídos menor número de correlações com apenas duas correlações positivas estimadas em função dos estágios de desenvolvimento dos Copepoda Harpacticoida: entre náuplios e adultos $(0,72)$ e entre náuplios e copepodito $(0,70)$. Copepoda Cyclopoida adulto mostrou correlação com Acarina $(0,78)$.

Em relação ao ambiente encrave (luminosidade intensa) além das correlações formadas pelos Copepoda Harpacticoida, houve a correlação de Annelida Polychaeta com copepodito $(0,77)$, com fêmeas ovígeras de Harpacticoida $(0,86)$, com Copepoda Cyclopoida adulto $(0,91)$ e com Ostracoda $(0,84)$.

No encrave onde a luminosidade é intermediária (profundidade de 2 metros) ocorreram apenas três correlações positivas, sendo duas entre Annelida Polychaeta com fêmeas ovígeras de Copepoda Harpacticoida $(0,96)$ e com adultos do mesmo grupo $(0,77)$, além daquela formada entre Harpacticoida adulto e suas fêmeas ovígeras $(0,91)$.

No encrave com luminosidade reduzida (profundidade de $3 \mathrm{~m}$ ) a meiofauna apresentou correlação positiva entre Annelida Polychaeta e Copepoda Cyclopoidea adulto $(0,86)$; e entre Copepodito e Harpacticoida adulto $(0,87)$.

\section{DISCUSSÃO}

A meiofauna dos ambientes recifais da porção sul de Tamandaré apresentou-se composta por oito taxa a nível de classe e ordem, pertencentes a três filos Nematoda, Annelida e Crustacea, não tendo sido possível identificar a nível específico. Destaca-se que a comparação dos taxa com outros ecossistemas nesse trabalho foi todo a nível de 
filo, classe ou ordem, devido a grande dificuldade, mesmo a nível internacional de se chegar a nível infragenérico.

Carvalho (1992) e Ayres, et al (1993) realizando trabalhos na baía de Tamandaré, em sua porção norte, e central onde ocorrem sedimentos do tipo areia média, encontraram 11 e 12 taxa, respectivamente, todos também identificados a nível de grupo (Classe, Filo).

Na baía de Suape, área localizada mais ao norte da baía de Tamandaré, Santos (1997) registrou 9 taxa. Fonseca-Genevois et al. (1996) registraram nas praias urbanas de Maria Farinha a Barra de Jangada 12 taxa. No istmo de Olinda, Bezerra (1994) registrou 21 taxa enquanto Victor-Castro (1998), registrou na restinga do Paiva 13 taxa.

No litoral norte de Pernambuco, Rocha (1991), registrou 13 taxa na margem sul da Ilha de Itamaracá. Na Coroa do Avião diversos autores (Fonseca-Genevois, et Al 1992; Lucena e Fonseca-Genevois; 1992; Esteves, 1995 e Pinto, 1998), registraram entre 8 e 12 taxa Almeida e Fonseca-Genevois (1999), registraram em Itapissuma (Canal de Santa Cruz) 13 taxa.

Pena (1998), trabalhando com meiofauna da laguna de Araruama, Rio de Janeiro identificou 14 taxa. Nessa área a salinidade varia de 12 a 65\%o, considerada eurihalina a hiperhalina. Silva (2001), trabalhando também com ambientes de alta salinidade (entre 55 a 120\%), Salina Diamante Branco, Galinhos, Rio Grande do Norte chegou a registrar 43 Gêneros de Nematoda distribuídos em 13 Famílias.

Apesar de diversas pesquisas empregarem metodologias de coletas diferentes, verifica-se que a meiofauna proveniente de vários ecossistemas do litoral pernambucano apresenta valores máximos variando de 2.916,8 a 20.358 ind. $/ 10 \mathrm{~cm}^{2}$.

Ao Norte do Canal de Santa Cruz foi registrado um valor máximo 20.458 ind. $/ 10 \mathrm{~cm}^{2}$, enquanto que na região central (Itapissuma) foi registrado 2.916 ind. $/ 10 \mathrm{~cm}^{2}$; na Coroa do Avião, 5.095 ind. $/ 10 \mathrm{~cm}^{2}$; na restinga do Paiva, $2.965,5$ ind. $/ 10 \mathrm{~cm}^{2}$; nas praias de Tamandaré, 5.301 ind./10 $\mathrm{cm}^{2}$ (Victor-Castro, 1998; Pena, 1998; Almeida e Fonseca-Genevois, 1999, Ribeiro, 1999).

Em regiões hipersalinas da lagoa de Araruama, Rio de Janeiro, Pena (1998), registrou um máximo de 9.464 ind. $/ 10 \mathrm{~cm}^{2}$, destacando que este valor diminui consideravelmente no período de inverno

Os ecossistemas marinhos mais produtivos do globo terrestre em termos de meiofauna são os estuários seguidos em ordem decrescente pela plataforma continental e mar profundo (Warwick e Prince, 1979; Renaud-Mornant et al 1984, apud Rocha et al 1998).

Assim, nos estuários encontram-se as maiores densidades de organismos meiofaunístico que em condições especiais chegam a atingir números na ordem de $10^{8}$ (Warwick e Prince, op cit) e isto, pode justificar a pequena quantidade da meiofauna registrado na área recifal de Tamandaré.

Por serem bastante numerosos, os organismos da meiofauna são de grande importância na rede trófica marinha, sendo necessário o aprofundamento de pesquisas nessa área. A meiofauna, especialmente Copepoda são conhecidos por servirem de alimento a uma grande variedade de predadores, especialmente peixes jovens (Coull, 1999; Silva, 2001). Bordiou (1999), registra para áreas da França que os copépodas

Trab. Oceanog. Univ. Fed. PE, Recife, 28(1): 47- 59, 2000. 
bentônicos são indispensáveis na dieta alimentar de numerosas espécies de peixes jovens.

Nematoda tem sido registrado dominando no conteúdo estomacal de várias espécies de peixes, e devido a sua fácil digestão não é registrado facilmente, porém se considera que Copepoda é a presa preferida de peixes jovens (Feller e Coull, 1995; Colobini et al, 1996, apud Coull, 1999)

Silva (2001), estudando a relação trófica de peixes no Canal de Santa Cruz, afirma que os peixes da Família Gerreidae alimentam-se preferencialmente de copépodos bentônicos ingerindo também copépodos planctônicos. Os Gobiidae tanto se alimenta de Copepoda quanto de Nematoda. Provavelmente, os Copepoda e Nematoda, grupos de maior abundância na área recifal de Tamandaré, entre na teia trófica de inúmeras espécies de peixes que habitam tal ecossistema. $\mathrm{O}$ pequeno número de espécimes encontrado, pode estar relacionado a intensa predação.

\section{REFERÊNCIAS BIBLIOGRÁFICAS}

ALMEIDA, Z. da S. de e FONSECA-GENEVOIS, V. Análise quali-quantitativo da meiofauna na região de Itapissuma-PE. Pesquisas em Foco, São Luiz: v. 7. n. 9. p.115-137. 1999.

AMARAL, F. D. Seções delgadas de algumas espécies de Scleractinios. Revista Biologica Brasílica. V. 6, n. Especial. p. 81-85. 1994/1995.

AMARAL, F. D. Sobre Favia leptophylla Verril 1868 (Cnidaria, Scleractnia) Iheringia. Série Zoologia, Porto Alegre. n. 73, p.117-118, 1992.

AYRES, C.F.J.; FONSECA-GENEVOIS, V.; GENEVOIS, B. Estabilidade meiofaunística na baía de Tamandaré; prospecções em uma estação controle para o Biomonitoramento litorâneo. In: V Congresso Nordestino de Ecologia. Natal, 1993, p.103. Resumos.

BARNES, R. D. Zoologia dos invertebrados. $4^{\mathrm{a}}$ ed. São Paulo: 1984, 1179p.

BERNSTEIN, B. B. et al. Spatial dispersion on benthic Foraminifera in the Abyssal Central Norte Pacific. Limnology and Oceanography. v.23 p.401-416, 1978.

BEZERRA, T. N. C. Introdução espaço-temporária da meiofauna do ístmo de Olinda-PE, com especial referência aos Nematoda. Recife: 1994, 66f. Dissertação (Mestrado em Oceanografia Biológica da UFPE).

BORDIOU, J. Y. Modalités de la prêdation des copépodes benthiques par les poissons. Vie et Milieu. v.49, n.4, p.301-308, 1999.

CARVALHO, et. al. Heterogeneidade espaço-temporal da meiofauna da baía de Tamandaré - PEBrasil. Biológica Brasilica, Recife, v.4, n. 1 e 2, 1992. p. 43-56.

CHAVES, P. T. C.; VANDEL, A. L. Aspectos da alimentação de Genidens genidens (Valenciennes) (Siluriformes, Ariidae) na baía de Guaratuba, Paraná. Rev. Bras. Zool. v.13, n.3, p. 669-675, 1996.

COÊLHO, P. A.; RAMOS-PORTO, M. Crustáceos da Região de Tamandaré, Estado de Pernambuco, Brasil. Boletim Técnico Científico do CEPENE, Tamandaré, v. 3, n. 1, p. 57-80, 1995.

COLOBINI,I.; BERTI, R.; NOCITA, A.; CHELAZZI,L. Foraging strategy of the mudskipper Periophthalmus sobrinus Eggert in a Kenyan mangrove. J. Esp. Mar. Biol. Ecol., n. 197, p.219-235, 1996. 
COULL, B. C. Role of meiofauna in estuarine soft-bottom habitats. Australian Journal of Ecology, n.24, p.327-343, 1999.

DYE A. H.; FURSTENBERG, J. P. An ecophysiological study of the meiofauna of the Swartkops estuary. II The mid faunal composition, distribution, seasonal fluctuations and biomass. Zool. Afr. v.13. n.1 p.19-32, 1978.

ESTEVES, A. M. Microdistribuição espacial da meiofauna na Coroa do Avião, Pernambuco. Dissertação (Mestrado) Mestrado em Biologia Animal, UFPE. Recife: 1995 $74 \mathrm{f}$.

FELLER, R. J. e COULL, B. C. Non-selective ingestion of meiobenthos by juvenile spot (Leiostomus xanthurus) and their daily ration. Vie Milieu, n.45, p.49-60, 1995.

FENCHEL, T. The ecology of micro and meiobenthos. Annual Review of Ecology and Systematics. v.9, p. 99-121, 1978.

FERREIRA, B. P.; MAIDA, M.; TEIXEIRA DE SOUZA, A. E. Levantamento inicial das comunidades de peixes recifais da região de Tamandaré, Boletim Técnico-Científico do CEPENE, v.3, n.1, p. 211-230, 1995.

FONSECA-GENEVOIS, V.; CARVALHO, I.; MARANHÃO, G. M. B.; LUCENA, W. Dados quantitativos sobre populações de Copepoda meiofaunística de ambientes recifais de Tamandaré. Resumos. Sociedade Nordestina de Zoologia, 1992.

FONSECA-GENEVOIS, V.; CARVALHO, I.; MARANHÃO, G. M. B.; LUCENA, W. Meiofauna paralitica/talassica do complexo de Tamandaré, litoral sul de Pernambuco. In: XIX Congresso Brasileiro de Zoologia e XII Congresso Latino-americano de Zoologia, 1992, Belém, Resumos .

FONSECA-GENEVOIS, V.; CARVALHO, I.; MARANHÃO, G. M. B.; LUCENA, W. Dados quantitativos sobre as populações de copepoda meiofaunísticos de ambientes recifais (Baia de Tamandaré, PE). In: IX Encontro de Zoologia do Nordeste. 1992. Resumos. Recife

FONSECA-GENEVOIS, V.; Ecologie des méio-et-mixofaunes d'une vasi're de l'estuari de la Lori: corrélations avec le milieu sedimentaire et ses eaux interstitielles. 1987. 366f. Tese ( Doutorado) Universidade de Nantes, França.

FONSECA-GENEVOIS, V.; JUNQUEIRA AYRES, C. Resultados preliminares sobre a meiofauna como vetor de impacto ambiental. Baía de Tamandaré (Litoral sul de Pernambuco). In: IX Encontro de Zoologia do Nordeste. 1992. Resumos. Recife.

FONSECA-GENEVOIS, V.; MARANHÃO, G. M. B. Composição meiofaunística, parâmetros físicos e sedimentológicos de beachrock , Pernambuco - Brasil. In: IX Semana Nacional de Oceanografia, 1996, Arraial do Cabo. Resumos.

GALVÃO, I. B.; LINS DA SILVA, N. M. Contribuição ao conhecimento do microzooplâncton da região de Tamandaré - PE - Brasil. In: XXI Congresso Brasileiro de Zoologia, 1996a . Porto Alegre. Resumos.

GALVÃO, I. B.; LINS DA SILVA, N. M. Dinâmica de população microfitoplanctônica e microzooplânctonica da região de Tamandaré - PE - Brasil. In: XXI Congresso Brasileiro de Zoologia, 1996b, Porto Alegre. Resumos.

GALVÃO, I. B.; LINS DA SILVA, N. M. Relações quantitativas entre os gêneros Lichophora melosiara e Gramathophora, na região de Tamandaré, PE, Brasil. In. VI Congresso Latinoamericano de Ciencias del Mar. 1995. Mar del Plata, Resumos. 
GOURBAUT, N.; RENAUD-MORANT, J.; HELLEÓUET, M-N. Biotopes et peuplements méiofaunistique des Petites Antiles (la marie - Golante, les Saintes, la Désirade), primiéres donnés. Bull. Mus. Nat. Hist. Paris, 4e. Ser. 3 Sect. A, p.419-431. 1985.

GUZMÁ, H. M.; OBANDO, V. L.; CORTÊS, J. Meiofauna associated with a Pacific coral reef in Costa Rica. Escuela de Biología and Cimar, Universidade de Costa Rica. San Pedro. Coral Reefs, v.6, p.107-112, 1987

LABOREL, J. Les peuplements de Madréporaires des côtes tropicales du Brésil. Marselha, Thèse A. O. Fac. Sci, 1967, 313p.

LIRA, L.; FONSECA-GENEVOIS, V. Interação biológicas da baía de Tamandaré, litoral sul de Pernambuco. Ciências Aquáticas. v. 4. p.85-108, 1998.

LUCENA, W. A.; FONSECA-GENEVOIS, V. Resultados preliminares sobre a meiofauna como vetor de impacto ambiental no Canal de Santa Cruz (Litoral norte de Pernambuco). In: IX de Zoologia do Nordeste. Recife: 1992, p.43.

MAIDA, M; FERREIRA, B. P. Coral reef of Brazil overview and field trip guide. In VIII International Coral Reef Syposium, Paraná, 1996.

MARANHÃO, G. M. B.; FONSÊCA-GENEVOIS, V. Copepoda Cyclopoidea da região infralitorânea da baía de Tamandaré: densidade e distribuição sazonal em ambientes recifais. In: V Congresso Nordestino de Ecologia, 1993. Resumos.

MARANHÃO, G. M. B.; FONSÊCA-GENEVOIS, V. Meiofauna de ambientes recifais (Tamandaré - PE). In: II Simpósio sobre Oceanografia. Resumos. 1991, São Paulo.

MARANHÃO, G. M. B.; FONSÊCA-GENEVOIS, V. Meiofauna parálica - Talássica do complexo Tamandaré, litoral sul de Pernambuco. In: XIX Congresso Brasileiro de Zoologia e XII Congresso Latino-Americano de Zoologia. Resumos, 1992, Belém.

MARE, M. F. Study of marine benthic community with especial reference to the microorganisms. J. Mar. Biol. Ass. U. K. V.25, p.517-544, 1942

MAYAL, E. M.; AMARAL, F. Ecomorfose em alguns escleractíneos da costa de pernambucana. Trab. Oceanogr. Univ. Fed. PE, v.21, p. 239-251, 1990.

MAYAL E.M.; BARONE, M. Bleaching in reef corals at Tamandaré in the southern coast of Pernambuco, Brazil. Bull. Mar. Sci. (no prelo).

MEDEIROS, C. G. Meiofauna de praia arenosa da ilha Anchieta. 1989. 376f. Dissertação (Mestrado) Mestrado em Zoologia da USP. São Paulo.

MOORE, C. G. The identification of faunal gradient and associations of meiobenthiquic copepods by multivariate. J. Mar. Biol. Assoc. U.K. v. 59, n. 3 p.725-736, 1979.

MOURA, R. T. Biomassa produção do fitoplâncton e alguns fatores ambientais na baía de Tamandaré, Rio Formoso, Pernambuco, Brasil. Dissertação. 1991, (Mestrado em Oceanografia da UFPE), Recife.

NASCIMENTO-VIEIRA, D. A. Macrozooplâncton recifal da baía de Tamandaré, Pernambuco - Brasil. Recife: 2000. 108 f. Tese (Doutorado) - Universidade Federal de Pernambuco. CTG. Oceanografia, 2000. Recife.

OLSON, T.; EROLSPM. B. Horizontal distribution of meiofauna within a small area, with special reference to Foraminifera. Zoon. v. 2, p.67-84, 1974.

PALMER, M.; GUST, G. Dispersal of meiofauna in a turbulent tidal creek. J. Mar. Res. n. 43, p.179-210, 1985. 
PENA, O. M. L. Hidróides do médio litoral de Tamandaré - Pernambuco. Taxonomia e sistemática. 1998, 66f. Monografia (Graduação) Curso de Ciências Biológicas da UFPE. Recife.

PINTO, T. K. de O. Estrutura da comunidade de meiofauna do banco de areia da Coroa do Avião - Itamaracá - PE - Brasil. 1998, 47f. Dissertação (Mestrado) Mestrado em Biologia Animal da UFPE, Recife.

PORTO NETO, F. F.; NEUMANN-LEITÃO, S.; NASCIMENTO-VIEIRA, D. A.; SILVA, A. P.; SILVA, T. A. ; MOURA, M. C. O. Zooplâncton recifal de Tamandaré-PE (Brasil) e a influência dos manguezais na sua biodiversidade e biomassa. In: MANGROVE 2000, Sustainable Use of Estuaries and Mangroves: Challenges and Prospects, 2000, Recife. Editora Universitária, 2000. v.1.

REANUD-DELLYSER, J. Recherches ecologiques sur la faune interstitialle des sable Basin d'Arcachon, Île de Bimini, Bahamas. Vie et Milieu. n. 5, p.1-157, 1963.

REISER, K.; AX, P. A. Meiofauanal (thiobios) limited to the anaerobic systematen of marine sand does not exist. Mar. Biol., v.54, p. 226-237, 1979.

RENAUD-MORNANT, ET AL. Estimations du rôle énergétique et dynamique spatiotemporalle du méiobenthos en millieu littoral: échantillonnage et méthodologie. Centro National de la Recherches Scientifique, 1984, 232 p.

RENAUD-MORNANT, J. G. N. Premiéres prospections méiofaunistiques en Guadeloupe. I. Les biotopes et leurs peuplements. Bull. Mus. Nat. Hist. Nat. Paris 4º S. 3 Sec. A. p. 10111034, 1981.

RIBEIRO, V. S. S. Relação dos fatores ambientais com a meiofauna na praia de Tamandaré, PE/Brasil. Dissertação (Mestrado). Recife, 1999. 76f. Dissertação (Mestrado). Mestrado em Biologia Animal da UFPE.

ROCHA, C. M. C. Meiofauna da margem da ilha de Itamaracá (PE), com especial referências aos Tardigrada. Recife, 1991. 264 f. Dissertação (Mestrado) Curso de Mestrado em Oceanografia Biológica.

ROCHA, C. M. C.; FONSÊCA-GENEVOIS, V.; VICTOR CASTRO, F. I. Distribuição espaço-temporal de Batlilipes pennaki Mareus, 1946 (Tardigrada, Heterotardigrada) na margem sul da Ilha de Itamaracá (Pernambuco, Brasil). Recife: 2000. Trab. Oceanog. . Univ. Fed. . PE, v. 28, n. 1, p. 35-46, 2000.

SANTOS, M. G. B. Efeitos da população por coliformes fecais sobre a comunidade da meiofauna nas praias urbanas de Pernambuco, Brasil. Recife: 1997, 87f. Monografia (Graduação) Curso de Ciências Biológica da UFPE.

SILVA, G. S. Prospecção do meiobentos mediolitorâneo da baía de Tamandaré, litoral sul de Pernambuco, com especial ênfase aos Acari. Recife, 1997 110f. Dissertação (Mestrado). Mestrado em Oceanografia Biológica da UFPE.

SILVA, N. R. R. da Distribuição espacial-temporária dos Nematoda livres em tanques de evaporação da Salina Diamante Branco. Natal- RN. Recife: 2001. Monografia (Graduação) Curso de Ciências Biológicas da UFRPE, 41f.

TEIXEIRA, A. L. Aspectos da caracterização ambiental da Coroa do Avião (ItamaracáPE). 1991, 113f. Monografia (Graduação) Curso de Ciências Biológica UFRN. Natal.

WARWICK, R. M.; PRICE, R. Ecological and metabolic studies on free living Nematodes from and estuarine mud-flat. Est. Mar. Sci., v. 9 p. 257-271, 1979. 